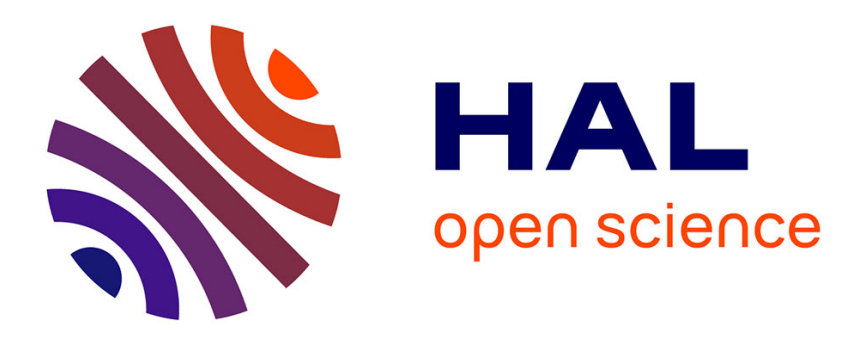

\title{
Some Results in Non-Linear Physics of Martensitic Transformation
}

\author{
O. Naimark, L. Filimonova
}

\section{To cite this version:}

O. Naimark, L. Filimonova. Some Results in Non-Linear Physics of Martensitic Transformation. Journal de Physique IV Proceedings, 1996, 06 (C1), pp.C1-65-C1-74. 10.1051/jp4:1996107 . jpa00254138

\section{HAL Id: jpa-00254138 https://hal.science/jpa-00254138}

Submitted on 1 Jan 1996

HAL is a multi-disciplinary open access archive for the deposit and dissemination of scientific research documents, whether they are published or not. The documents may come from teaching and research institutions in France or abroad, or from public or private research centers.
L'archive ouverte pluridisciplinaire HAL, est destinée au dépôt et à la diffusion de documents scientifiques de niveau recherche, publiés ou non, émanant des établissements d'enseignement et de recherche français ou étrangers, des laboratoires publics ou privés. 


\section{Some Results in Non-Linear Physics of Martensitic Transformation}

O. Naimark and L.V. Filimonova

Institute of Continuous Media Mechanics of the Russian Academy of Sciences 1, Acad. Korolev str., 614061, Perm, Russia

\footnotetext{
Abstract.Statistical thermodynamics of martensitic transformation is developed in the frame of the self-consistent field approximation. The accommodation mechanisms of the parent phase to the martensitic variant growth are described by the three order parameters. The latters reflect the concentration and the orientation of the martensitic phase and the dislocation density at the interphase boundaries. Statistical model allowed to explain the superelasticity and shape memory effects and to propose the phenomenological description of deformation properties caused by martensitic transformation. Taking into account the typical nonlinearities for the order parameters given by statistical model the wave nature of the martensitic phase growth is analysed.
}

\section{REVERSIBLE PLASTICITY OF ALLOYS DUE TO THE SOLID-SOLID PHASE TRANSFORMATION}

\section{$1.1 \quad$ Introduction}

Usual plasticity is understood as the ability of solid to the irreversible deformation and it is well known that the developed plasticity of crystals is realized due to the displacement of defects in the crystal lattice. For some crystals the internal forces arise due to the twinning or thermo-elastic martensitic transformation and it is the reason to promote the reverse motion of defects after unloading. This process may be qualified as a reversible plasticity [1]. In the modern plasticity theory irreversible deformation is considered as the dislocation movernent. A dislocation displacement occurs due to the apply of external forces when the latters exceed some yield value. After the unloading the stresses caused by the interaction between dislocations are as rule not enough for the surmounting of the yield barrier and deformation created by dislocation displacement becames irreversible. Another situation is realized when deformation is caused by the phase transformation in crystals. Plasticity in this case is developed due to the displacement of the so-called transformation dislocations and their replacement determines the interphase boundary movement. Under special conditions the internal forces caused by a new phase generation may exceed significantly the yield stress of the dislocation start that leads to return movement of dislocation for a long distance.

\subsection{Accommodation Mechanisms of Martensitic Transformations}

It is of important to establish the main mechanisms of accommodation on the nucleation and growth of the martensitic phase because these mechanisms determine the number and types of the indepen- 
dent variables playing the role of the order parameters. Specific mechanism of accommodation is caused by the form of the transformation strain which satisfies the compactibilty condition under elastic behaviour of martensitic and austenitic phase. It is the so-called deformation with invariant plane giving the polysynthetic twin structure of self-accommodated variants. In this case a new independent variable is transformation strain $m_{i k}$ with the following type of the symmetry:

$$
m_{i k}=\frac{1}{2} m\left(\nu_{i} \tau_{k}+\tau_{i} \nu_{k}\right)
$$

where $m$ is a parameter of the lattice deformation due to the transformation; $\vec{\nu}$ and $\vec{\tau}$ are unit vectors which determine the invariant plane orientation and the shearing direction.

The second mechanism of accommodation corresponds to the possibility to realize the thermo-elastic equilibrium state of martensitic and austenitic phases when the complete coherensity is provided between a new phase and a parent one. The solid state is characterized in this case by a scalar parameter of the martensitic concentration $c=V_{m} / V_{n}$ and the tensor of the transformation strain $\epsilon_{i k}^{t r}$. Here $V_{n}=V / n$ is specific volume corresponding to one variant, $V_{m}$ is the volume of the martensitic variant. Transformation strain tensor is given in this case by formula

$$
\epsilon_{i k}^{t r}=\frac{1}{2} c m\left(\nu_{i} \tau_{k}+\tau_{i} \nu_{k}\right)
$$

The next additional parameter may be introduced in the theory for the description of deformation at the interphase boundary due to the breaking of coherencity. Apparently a symmetry group of this parameter coinsides with a symmetry group of deformation with invariant plane

$$
s_{i k}=\frac{1}{2} s\left(\nu_{i} \tau_{k}+\tau_{k} \nu_{i}\right)
$$

where $s$ is the value of plastic deformation. The nature of this deformation is the generation of transformation dislocations at the interphase boundary that provides the propagation of the transformation front and accommodation conditions.

\section{STATISTICAL MODEL AND CONSTITUTIVE EQUA- TIONS}

\subsection{Statistical Model}

The next step of our theoretical approach is an attempt to apply a statistical thermodynamic method to the description of typical responses of solid with martensitic inclusions taking into consideration the interaction between martensitic variants due to the appearance of the internal stress field caused by a new phase nucleation and growth.

The set of the independent thermodynamic variables includes: transformation strain tensor $m_{i k}$ with the invariant plane type of symmetry, scalar parameter of the volume concentration of martensite $c$ and the plastic strain tensor $s_{i k}$ caused by dislocation density at the interphase boundary. Statistical theory is developed in the frame of the one-particle approximation [2] when we consider the thermodynamic ensemble of the same martensitic variants.

Macroscopic order parameters $\bar{\epsilon}_{i k}^{t r}, \bar{c}$ and macroscopic plastic strain $s_{i k}$ caused by the new phase development are determined due to the averaging of the corresponding microscopic tensors with some distribution function.

$$
\bar{\epsilon}_{i k}^{t r}=\left\langle\epsilon_{i k}^{t r}\right\rangle=\bar{c} \frac{1}{2} g\left(n_{i} t_{k}+t_{i} n_{k}\right), \quad \bar{c}=\left\langle\frac{V_{m}}{V_{n}}\right\rangle, \quad S_{i k}=\left\langle s_{i k}\right\rangle .
$$

Normalizing condition for $\bar{\epsilon}_{i k}^{\text {tr }}$ follows from the obvious relations for complete oriented and disoriented variants: $g=m$ if $\langle\vec{\nu} \vec{n}\rangle=1$ or $g=0$ if $\langle\vec{\nu} \vec{n}\rangle=0$. An important feature of the thermo-elastic 
martensitic transformation is the equilibrium character of the latter [3]. It means that in some cases the distribution function may be taken as the Gibbs function

$$
W(s, \vec{\nu}, \vec{\tau})=Z^{-1} \exp \left(-\frac{E}{T}\right), Z=\int \exp \left(-\frac{E}{T}\right) d \Omega d c,
$$

where $E$ is the energy of volume $V_{n}$ which corresponds to single variants; $T$ is temperature measured in the energetic units; $Z$ is the normalizing parameter; $\Omega$ is the orientation space of variants. For the determination of energy $E$ the self-consistent field method was used in the variant that was developed for statistical analysis of the system behaviour under the phase transformation. The expression for energy $\mathrm{E}$ was obtained in the frame of the mean field approximation when the interaction energy between martensitic variants $-H_{i k} \epsilon_{i k}^{t r}$ is taken as a special form of the effective stress field $H_{i k}=$ $\gamma \sigma_{i k}+\lambda \bar{\epsilon}_{i k}^{t r}$

$$
E_{V_{n}}=E_{0} V_{n}+V_{n}\left(-H_{i k} \epsilon_{i k}^{t r}+\frac{1}{2} \bar{\mu} m^{2} c(1-\bar{c})\right)-B V_{m}
$$

Here $E_{0}=1 / 2 \lambda \bar{\epsilon}_{i k}^{t r}+1 / 2 \bar{\mu} m^{2} \bar{c}^{2}$ is the macroscopic term; $1 / 2 \bar{\mu} m^{2} c(1-\bar{c}) V_{n}$ is the own energy of the martensitic variant; $B$ is the difference in free chemical energy of martensitic and austenitic phases; $\gamma$ and $\lambda$ are mean field parameters.

Constant $\bar{\mu}$ is the first term in expansion for the tensor function $M_{i k l m}$ that is determined by the shape of martensitic variants

$$
M_{i k l m}=\bar{\mu} \delta_{i k} \delta_{l m}+\bar{\mu}_{1}\left(\bar{\epsilon}_{i l}^{t r} \delta_{k m}+\bar{\epsilon}_{k m}^{t r} \delta_{i l}\right)
$$

The average of $\epsilon_{i k}^{t r}$ and $c$ in space of the possible orientation and concentration gives the selfconsistent equations for $\overline{\epsilon_{i k}}$ and $\bar{c}$

$$
\begin{aligned}
\epsilon_{i k}^{\overline{t r}} & =\int \epsilon_{i k}^{i r} Z_{0}^{-1} \exp \left(-\frac{E_{V_{n}}}{T}\right) d c d \Omega \\
\bar{c} & =\int c Z_{0}^{-1} \exp \left(-\frac{E_{V_{n}}}{T}\right) d c d \Omega .
\end{aligned}
$$

The average procedure was realized for the shear loading assuming that the transformation strain has only shear components. The components of the stress tensor may be represented in this case as $\sigma_{i k}=1 / 2 \sigma_{12}\left(n_{i} t_{k}+t_{i} n_{k}\right)$. The part of the free energy $F^{t r}$ caused by the new phase generation has the following form

$$
F^{t r}=\frac{1}{2} \lambda \bar{\epsilon}_{i k}^{t r 2}+\frac{1}{2} \bar{\mu} m^{2} \bar{c}^{2}-n T \ln Z_{0}^{\prime}
$$

where $Z_{0}^{\prime}=\int \exp \left(-E_{V_{n}}^{\prime} / T\right)$ and $E_{V_{n}}^{\prime}$ depend only on "microscopic" part.

It is obvious that the equilibrium conditions

$$
\frac{\partial F^{t r}}{\partial \bar{\epsilon}_{i k}^{t r}}=0, \quad \frac{\partial F^{t r}}{\partial \bar{c}}=0
$$

coinside with the self-consistent equations. The results of numerical solutions of the self-consistent equations are presented in Fig. 1 for the followig dimensionless variables

$$
\theta=T /\left(1 / 2 \bar{\mu} m^{2} V_{n}\right), \quad \Sigma=\sigma_{12} /\left(1 / 2 \bar{\mu} m^{2}\right), \quad \bar{g}=g / m,
$$

combined with the corresponding free energy dependences. The dependences of concentration on temperature for different stresses show the existence of critical temperatures for the low-temperature phase nucleation which are shifted in the high-temperature range for the stress load. At the temperatures below critical the concentration dependences demonstrate the metastability of parent phase that doesn't exclude the existence of the austenitic phase below critical points for the high rate of the temperature decrease. For the order parameter $\bar{g}$ solution gives the symmetrical picture for $\Sigma=0$ in some width of the metastability range $\theta_{g}^{f}<\theta_{g}^{c}<\theta_{g}^{s}$. These temperatures are shifted in the high temperature field under the stress action. Symmetrical picture of $\bar{g}(\theta)$ at $\Sigma=0$ reflects the degeneration of the preferential domain orientation in the stress free state. 

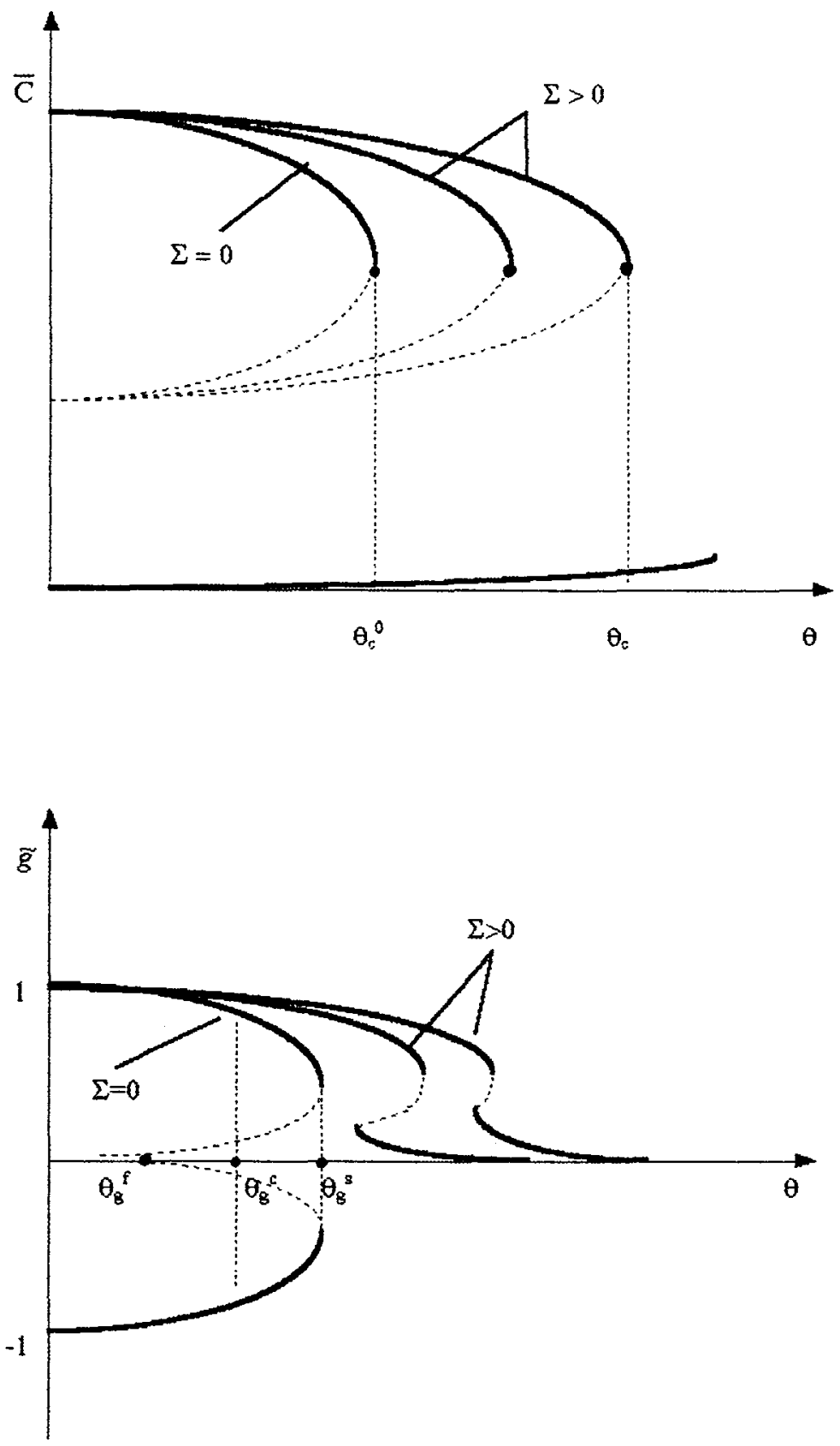

Figure 1. Solutions of the self-consistent equations. 


\section{DEFORMATION PROPERTIES UNDER MARTEN- SITIC TRANSFORMATION}

\subsection{Constitutive Equations for Thermo-Elastic Martensitic State}

For the study of the deformation properties of alloys with the martensitic type transformation we introduce the total free energy $F$. This quantity is made up of the phase transformation part of the free energy $F^{t r}$ and the normal elastic energy [2]:

$$
F=F^{t r}+\mu\left(\epsilon_{i k}-1 / 3 \epsilon_{l l} \delta_{i k}\right)^{2}+K \epsilon_{l l}^{2} / 2
$$

where $\epsilon_{i k}$ is the macroscopic strain tensor; $\mu$ and $K$ are the shear and bulk moduli. Since $F$ contains the stress, $\sigma_{i k}$, in its explicit form, but not strain, it is convenient to go over to the thermodynamic potential $\Phi=F-\sigma_{i k} \epsilon_{i k}$, for which the role of independent variables is played by the components of the $\sigma_{i k}$ tensor. Expressing in (13) $\epsilon_{i k}$ in terms of $\sigma_{i k}$ the strain tensor is obtainable here by differentiating of the potential $\Phi[3]$ :

$$
\epsilon_{i k}=-\frac{\partial \Phi}{\sigma_{i k}}=\frac{1}{2 \mu}\left(\sigma_{i k}-\frac{1}{3} \sigma_{l l} \delta_{i k}\right)+\frac{1}{9 K} \sigma_{l l} \delta_{i k}+\epsilon_{i k}^{t r} .
$$

Here we have used the determination for $\epsilon_{i k}^{t r}$ which follows from (8). The formula (14) and solution of the self-consistent equations for the order parameters $\epsilon_{i k}^{t r}$ and $C$ contain the interpretation of the shape memory effect and superelasticity.

\subsection{Shape memory effect}

Superelasticity and shape memory effect are more impressive phenomena of the reversible plasticity. Superelasticity is observed when in strained alloys the large deformation disappears after unloading. Shape memory effect is the phenomenon when the specimen being deformed plastically restores the initial shape after the heating. These effects are observed due to the thermo-elastic martensitic transformation development in the temperature range of the co-existence of two phases. Let's to consider more detailed interpretation of the shape memory effect using the results of the solution of self-consistent equations presented in Fig.2 coupled with the scheme of the typical experiment which illustrates this effect. Due to the cooling from temperature $\theta_{1}$ in the range $\theta<\theta_{g}^{s}$ for the stress free specimen the system of the coherent domains is created with order parameters $g_{2}$ and $-g_{2}$. The following loading transfers the system into the state $g_{3}$ that is realized by the jump-like reorientation of domains having the contrary orientation to the external stress field. After the unloading $(3 \rightarrow 4)$ the elastic part of deformation disappears but the deformation caused by orientation $g_{4}$ is kept and only the heating $(4 \rightarrow 1)$ restores the initial state.

\subsection{Superelasticity effect}

Superelasticity phenomenon is caused by the appearance of martensitic variants due to the stress load at the temperature $\theta>\left(\theta_{0}^{c}, \theta_{g}^{c}\right)$, when the martensitic phase is stable only in the external stress field. The increase of the external stress leads to grow of martensitic inclusions and the latters grow less under the unloading. This effect reminds the elastic twinning but for superelasticity the driving force acting on the transformation dislocations depends on temperature.

The formula (14) describes the transformation-elastic effects. It follows, in particular, from the fact that a change in the degree of orientation of the martensitic variants and their concentration leads to spontaneous deformation (orientation striction). The isothermal loading of alloys in this temperature range induces, upon the attainment of a certain value of $\Sigma_{m}$, the jump of deformation " "orientational flow" - as the result of collective behaviour in the system of martensitic variants. 

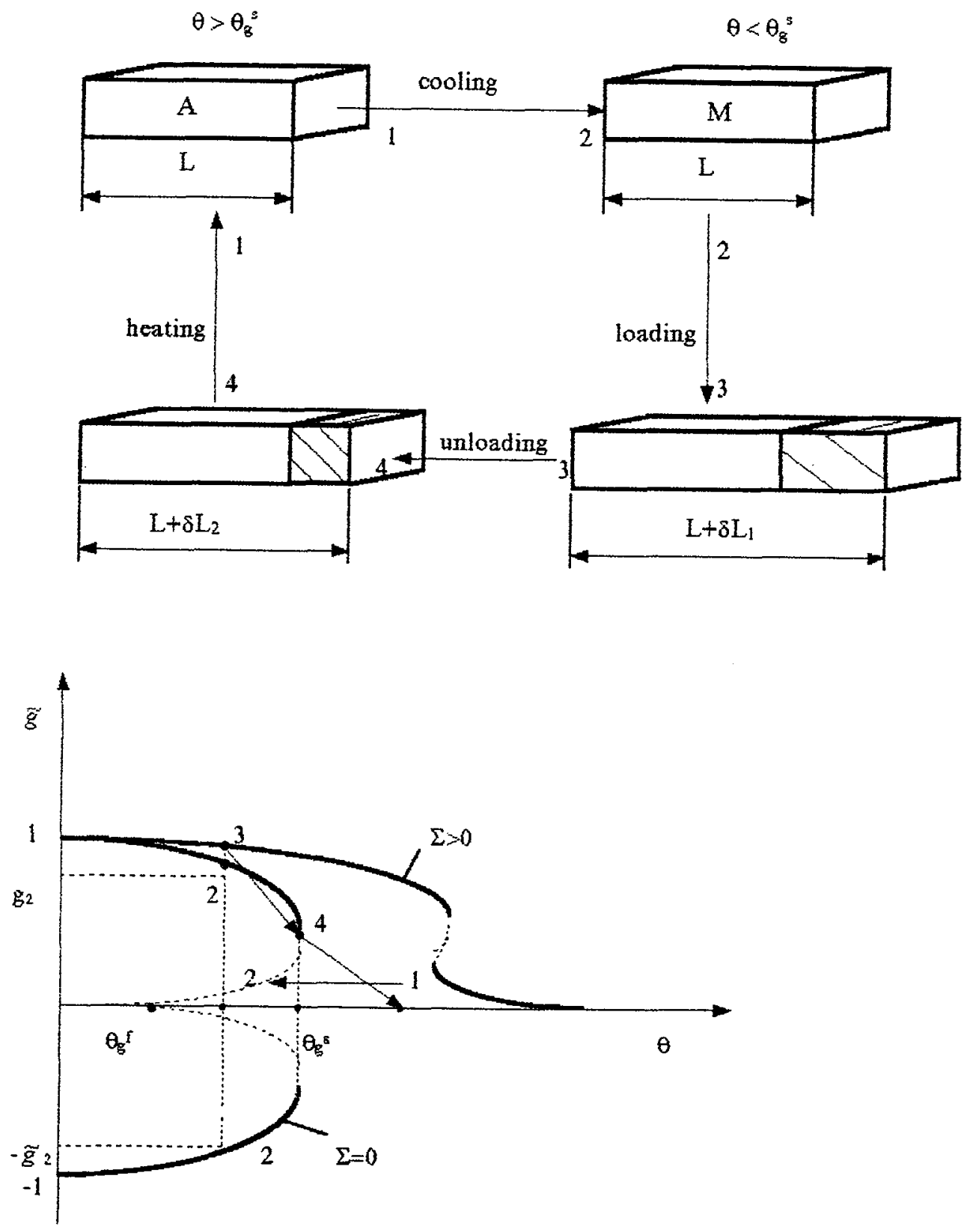

Figure 2. Interpretation of the shape memory effect. 


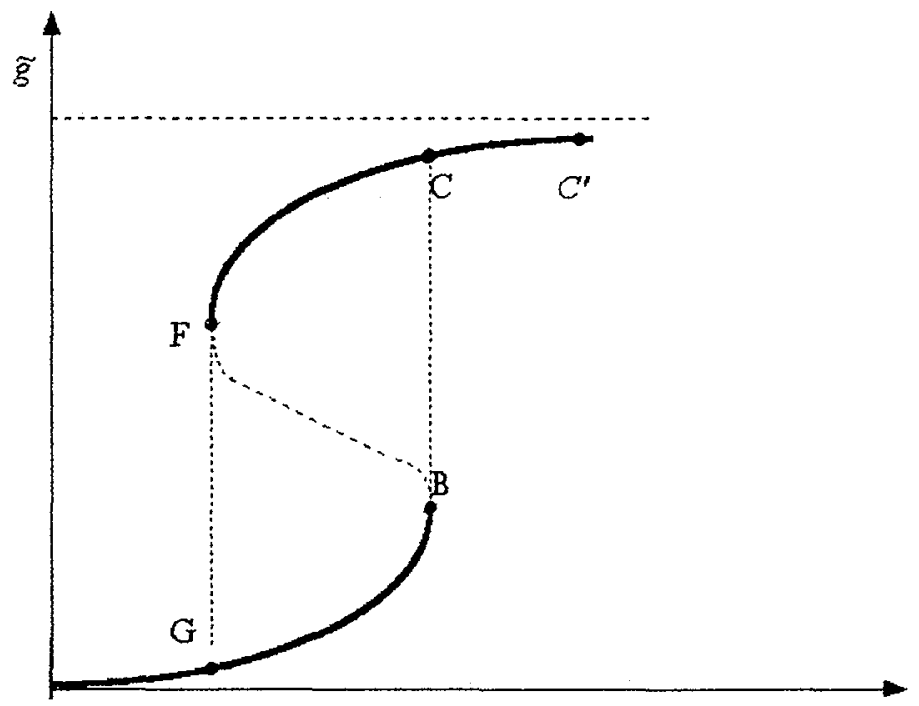

$\Sigma$

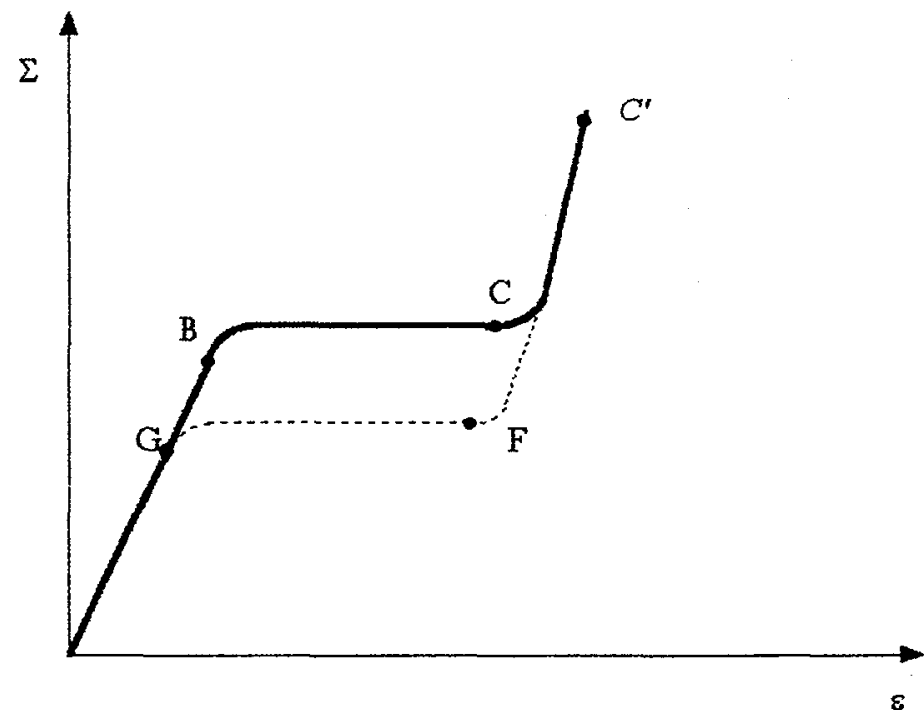

Figure 3. Deformation property under martensitic transformation 
"Orientational flow" corresponds to the horizontal plateau in curve in Fig.3. Point $B$ is the start of the martensite inclusion nucleation and the corresponding stress level $\Sigma_{m}$ is caused by the penetration depth in the metastability interval $\Sigma_{0}<\Sigma<\Sigma_{m}$. This penetration is the necessity to surmount the energy barrier for the interphase boundary movement a.t the given temperature. The stress increase transfers the bulk of specimen in martensitic phase $(B \rightarrow C)$ with the subsequent elastic deformation of the latter $\left(C \rightarrow C^{\prime}\right)$. The branch $C^{\prime} F$ presents the elastic unloading of martensitic phase and the portion $F G$ corresponds to the nucleation and growth of the austenitic phase under the condition of the new orientational transition. But the qualitative character of transition is maintained and needs the some decrease of the transition stress below $\Sigma_{0}$ for the growing of the austenitic variants in martensite.

\section{THE PHENOMENOLOGY OF MARTENSITIC TRANSFORMATION}

\subsection{The Landau free energy expansion}

Statistical model allowed to establish characteristic solid responses on stress and temperature under martensitic transformation. It is important because in the attempts to apply the Landau approach [4] to the description of martensitic transformation some formal analogies were used $[5,6,7]$. But statistical consideration reflects the significant role of stresses and the interaction between variants as the specfic features of thermo-elastic transformation and established the type of order parameters. According to these results we shall introduce the expansion for the transformation part of the free energy $F^{t r}$. As it is obvious the minimal power of the expansions for the tensor $g_{i k}=1 / 2 g\left(n_{i} t_{k}+t_{i} n_{k}\right)$ and scalar $c$ order parameters are the sixth and the fourth degree correspondingly

$$
F^{t r}=A_{1} \mathbf{g}^{2}-B_{1} \mathbf{g}^{4}+C_{1} \mathbf{g}^{6}-D \sigma \mathbf{g}+A_{2} c^{2}-B_{2} c^{3}+C_{2} c^{4}
$$

where $A_{1}=A_{1}^{\mathrm{o}}\left(T-T_{1}\right), A_{2}=A_{2}^{\mathrm{o}}\left(T-T_{h}\right) ; B_{1}, C_{1}, \ldots C_{2}$ are the phenomenological parameters depending on concentration $c$ and invariants of tensor $\mathrm{g} ; T_{1}$ and $T_{h}$ are characteristic temperatures (Fig.1) connected by relations:

$$
T_{2}=T_{1}+\frac{1}{3} \frac{B_{1}^{2}}{A_{1}^{0} C_{1}}, T_{0}=T_{1}+\frac{1}{4} \frac{B_{1}^{2}}{A_{1}^{0} C_{1}}, T_{c}=T_{h}+\frac{9}{32} \frac{B_{2}^{2}}{A_{2}^{0} C_{2}}, T_{c} \geq T_{2} .
$$

The free energy form (15) gives the description of typical responses and allows to study some nonlinear effects observed in experiments.

\subsection{Kinetic equations for the order parameters}

The nonlinear aspects of the martensitic phase development were investigated in $[8,9]$ taking into consideration the wave structure of the interphase front. Keeping in mind to consider the spatially inhomogeneous distribution of martensitic phase we include in the expression (15) the terms describing the possible inhomogeneity of order parameters

$$
\tilde{F}^{t r}=F^{t r}-\frac{1}{2} \rho_{g}(\nabla \mathbf{g})^{2}-\frac{1}{2} \rho_{c}(\nabla c)^{2} .
$$

This type of free energy approximation (long-wave approximation) is usually called the GinzburgLandau expansion [10]. Kinetic equations may be obtained using the evolution inequality

$$
\frac{\delta F^{t r}}{\delta t}=\frac{\delta \tilde{F}^{t r}}{\delta \mathbf{g}} \dot{\mathbf{g}}+\frac{\delta \tilde{F}}{\delta c} \dot{c} \leq 0,
$$


where the driving forces $\delta \tilde{F} / \delta \mathbf{g}, \delta \tilde{F} / \delta c$ are given by variational derivatives so that for $\mathbf{g}$ and $c$ the motion equations follow

$$
\begin{aligned}
& \dot{\mathrm{g}}=-\Gamma_{g} \frac{\partial F^{t r}}{\partial \mathrm{g}}+\frac{\partial}{\partial x_{l}}\left(\chi_{g} \frac{\partial \mathrm{g}}{\partial \mathrm{x}_{\mathrm{l}}}\right), \\
& \dot{c}=-\Gamma_{c} \frac{\partial F^{t r}}{\partial c}+\frac{\partial}{\partial x_{l}}\left(\chi_{c} \frac{\partial c}{\partial x_{l}}\right) .
\end{aligned}
$$

Here $\Gamma_{g}$ and $\Gamma_{c}$ are kinetic coefficients; $\chi_{g}=\rho_{g} \Gamma_{g}, \chi_{c}=\rho_{c} \Gamma_{c}$.

\section{THE WAVE NATURE OF THE MARTENSITIC PHASE DEVELOPMENT}

\subsection{Some experimental results}

The interpretations of the phase transformation kinetics are usually connected with the surmounting of the energy barriers due to the appearance of thermodynamical driving forces [11]. Kinetic mechanisms of the martensitic phase growth were examined in $[8,9]$ and it was established that the rate of martensitic crystal growth exceeds both the longitudinal and transverse elastic waves.

The high rate of interphase boundary must be accompanied by supersound dislocation movement. For the continuum model the exceeding of the sound rate is not correct taking into account the property of ordinary dislocations [12]. But for the real crystals with discrete structure it seems to be possible. The same situation appears under analysis of shock wave propagation in solid [13]. Probably for the first time Machlin ant Cohen [8] formulated the wave approach in the problem of martensite growth. Mayers and Mur [9] considered the interphase boundary movement as the wave process. In the course of the latter the specific type of dislocations appears and the interphase boundaries behind of the propagating wave front are created. Martensite growth was considered as the propagation of two waves of deformation. The first wave describes the lattice deformation, the second one corresponds to the deformation with invariant plane. Using the ideology developed by Machlin and Cohen, Mayers postulated the longitudinal character of the radial waves. Materials subject to the radial excitation play the role of the nuclei of the second order for the initiation of the shear (transverse) waves which are propagated in the perpendicular direction.

\subsection{The wave model of the martensitic phase growth}

The review of papers having the relation to the spontaneous martensitic transformation shows that the wave approach is perspective for the development of the microscopic theory. In $[14,15]$ the martensite growth was analysed on the basis of theory of shock wave propagation. It was admitted the powerfull of shock wave generation that allowed to explain the supersound rate of martensitic phase growth. But the estimation of stresses arising in solid showed the values exceeding the theoretical strength limit.

The main goals of this approach is to establish the mechanism and nature of wave generation and as the consequence to explain specific crystallographic signs of the martensitic phase generation.

Experiments [16] supported by the results of the developed statistical model revealed the metastability of parent phase in the range $\theta<\theta_{c}$ (Fig.2). The wave of displacement in interphase area provides the surmounting over the energetic barrier. The value of the latter depends on the depth of penetration in metastability region due to the overcooling. For the system with two metastable states which are divided by energetic barrier the solution in the form of trigger switching waves are available [17]. Considering the stress free state $(\mathbf{g}=0)$ kinetic equations $(20)$ may be represented in the form of so-called "switch approximation" [14].

$$
\frac{d c}{d t}=-\frac{1}{\tau_{c}} c\left(c-c_{t}\right)\left(c-c_{m}\right)+\chi_{c} \Delta c
$$


where $\tau_{c}=\Gamma_{c}^{-1}$, parameters $c_{t}$ and $c_{m}$ are given by the relations: $c_{t} c_{m}=2 A_{2} / C_{2}, c_{t}+c_{m}=-4 B_{2} / 3 C_{2}$. Equation (21) has the solution in the form of stationary wave $c(\xi)=c\left(x-V_{c} t\right)$

$$
\left.c=1 / 2 c_{m}\left(1-t h\left(2 \xi l_{c}\right)^{-1}\right)\right) ; l_{c}=4 / c_{m}\left(2 \chi_{c} \tau_{c}\right)^{1 / 2}
$$

The relations (22) belong to the class of invariant group solutions. The rate of wave propagation $V_{c}$ is determined by the overcooling degree and continuously increases with the depth of penetration in metastability area $V_{c}=\left(\chi_{c} / 2 \tau_{c}\right)^{1 / 2}\left(c_{m}-2 c_{t}\right)$. This result explains the explosion-like growth of martensite inclusions which is accompanied by brittle fracture of specimen for the high speed overcooling of austenite that was observed by Chumljakov's experiments [16]. We considered only concentration waves but in real materials due to the appearance of structural stresses the tensor part of wave solution of equation (19) is realized. The interaction between the concentration and orientation waves ( $c$ and $\mathbf{g}$ ) leads to the sharp increase of the rate of interphase front propagation. The solution for two independent variables $c$ and $\mathbf{g}$ describes the preliminary lattice excitation into the concentration front preceeding to the change of lattice symmetry and the following generation of transverse waves.

\section{Acknowledgments}

The present paper was finished during the author stay as a visiting professor at the Laboratory of Physics and Mechanics of Materials of the University of Metz. The author is kindly indebted to Professor M.Berveiller for their invitations and very helpful discussions and gratefully acknowledges support from C.N.R.S.

\section{References}

[1] Boiko V.S., Garber R.I., Kosevich A.M., Reversible plasticity in crystals (Nauka, Moscow, 1991) p.280 (in Russian)

[2] Naimark O.B., Silbershmidt V.V., Eur.J.Mech., A/Solids, 10, n.6 (1991) 607-619

[3] Landau L.D., Lifshitz E.M., Elasticity theory (Nauka, Moscow, 1965) p.203 (in Russian)

[4] Landau L.D., Khalatnikov L.M., Sov. Phys.Dokl., 96 (1954) 469-474 (in Russian)

[5] Falk F., J. de Physique, 43, n.12 (1982) 3-14

[6] Gooding R.J., Bales G.S., J. de Physique, 1 (1991) 4-59

[7] Bach G.R., Martensite (ASM, 1992) pp.125-174

[8] Machlin E.S., Cohen M., Trans.AIME, 191 (1951)1019-1029

[9] Mayers M.A., Acta Metall., 28 (1980) 757-770

[10] Landau L.D., Lifshitz E.M., Statistical Physics (Nauka, Moscow, 1978) p.576 (in Russian)

[11] Patoor E., Eberhardt A., Berveiller M., Arch.Mech., 40, 5-6 (1988) 775-794

[12] Kosevich A.M., Dislocation in the elasticity theory (Naukova Dumka, Kiev, 1978) p.220 (in Russian)

[13] Zel'dovich Ya.B., Raiser Ju.P. Physics of shock waves and high temperature hydrodynamic phenomena (Nauka, Moscow, 1966) p.686 (in Russian)

[14] Kashenko M.P., The wave model of martensite growth for $\gamma-\alpha$ transformation in iron-based alloys (UIF "Nauka", Ekaterinburg, 1993) p.224 (in Russian)

[15] Lokshin F.L.,Scientific reports of High School, 2 (1957), 205-208 (in Russian)

[16] Chumljakov Ju.I., Private communication.

[17] Yachno V.G. Biophysics, 21, 8 (1976), 547-550 (in Russian) 